\title{
Does a woman's educational attainment influence in vitro fertilization outcomes?
}

\section{Citation}

Mahalingaiah, Shruthi, Katharine F. Berry, Mark D. Hornstein, Daniel W. Cramer, and Stacey A. Missmer. 2011. “Does a Woman's Educational Attainment Influence in Vitro Fertilization Outcomes?" Fertility and Sterility 95 (8) (June): 2618-2620. doi:10.1016/j.fertnstert.2011.05.015.

\section{Published Version}

doi:10.1016/j.fertnstert.2011.05.015

\section{Permanent link}

http://nrs.harvard.edu/urn-3:HUL.InstRepos:27336530

\section{Terms of Use}

This article was downloaded from Harvard University's DASH repository, and is made available under the terms and conditions applicable to Other Posted Material, as set forth at http:// nrs.harvard.edu/urn-3:HUL.InstRepos:dash.current.terms-of-use\#LAA

\section{Share Your Story}

The Harvard community has made this article openly available.

Please share how this access benefits you. Submit a story.

\section{Accessibility}




\title{
Does a woman's educational attainment influence in vitro fertilization outcomes?
}

\author{
Shruthi Mahalingaiah, M.D. ${ }^{a}$, Katharine F. Berry, M.S. ${ }^{a}$, Mark D. Hornstein, M.D. ${ }^{a}$, Daniel W. \\ Cramer, M.D., Sc.D. ${ }^{a}$, and Stacey A. Missmer, Sc.D. ${ }^{a, b, c}$ \\ aDepartment of Obstetrics, Gynecology, and Reproductive Biology, Brigham and Women's \\ Hospital and Harvard Medical School, Boston, Massachusetts \\ bepartment of Epidemiology, Harvard School of Public Health, Harvard University, Boston, \\ Massachusetts \\ 'Channing Laboratory, Department of Medicine, Brigham and Women's Hospital and Harvard \\ Medical School, Boston, Massachusetts
}

\begin{abstract}
The association between educational level and cycle outcomes was quantified by applying multivariable logistic and linear regression within a prospective cohort of 2,569 women commencing their first in vitro fertilization (IVF) cycle. Although a woman's educational attainment was not associated with the likelihood of implantation failure, chemical pregnancy, spontaneous abortion, or live birth, the odds of cycle cancellation before egg retrieval were $40 \%$ lower among those with an college degree and $48 \%$ lower among those with graduate school attendance compared with women who had no college degree, suggesting that educational attainment is inversely associated with the likelihood of cycle cancellation.
\end{abstract}

\section{Keywords}

ART; education; IVF outcomes; maternal

A strong relation between maternal educational attainment and a child's cognitive development has been demonstrated (1), so within the field of assisted reproductive technology (ART), studies addressing the influence of maternal education have also largely focused on the cognitive, motor, and developmental outcomes in the children $(2,3)$. It remains unclear, however, whether a woman's education influences the outcome of in vitro fertilization (IVF) treatment itself.

Women who delay childbearing until the completion of longer educational training may comprise a large segment of the IVF patient population and may drive the observation of an age-confounded inverse relation between educational level and successful IVF outcome. Conversely, it may be that women with higher educational attainment are more likely to correctly perform the stimulation protocol, thus improving their likelihood of treatment success. If this association holds true, there may be potential for maximizing IVF success

Copyright @2011 American Society for Reproductive Medicine, Published by Elsevier Inc.

Reprint requests: Shruthi Mahalingaiah, M.D., Department of Obstetrics, Gynecology, and Reproductive Biology, Brigham and Women's Hospital, 75 Francis Street, Boston, Massachusetts 02115 (smahalingaiah@ partners.org).

S.M. has nothing to disclose. K.F.B. has nothing to disclose. M.D.H. is on the Medical Advisory Board of WIN Fertility and a contributor to UpTo-Date. D.W.C. has nothing to disclose. S.A.M. has nothing to disclose. 
with improved educational interventions to minimize the risk for self-administered protocol errors. We investigated the association between a woman's educational status, independent of age, and her IVF outcome within a large cohort of women commencing their first IVF cycle at one of three clinics in the greater Boston area between 1994 and 2003. Further details of the study enrollment have been described previously elsewhere (4).

This study was approved by the institutional review boards of Brigham and Women's Hospital and Harvard School of Public Health. Married couples, literate in the English language, who were undergoing their first in vitro fertilization or intracytoplasmic sperm injection (IVF-ICSI) cycle were eligible to participate. We excluded women using donor gametes or gestational carriers, and those undergoing gamete or zygote intrafallopian transfer, freeze-all cycles, or intrauterine insemination conversion to IVF. Before the start of the cycle, the couples completed questionnaires that elicited their educational history, demographic, and lifestyle information. The three clinic sites, including an academic center, an academically-affiliated private practice, and a suburban private clinic, may represent slightly different patient populations.

The women were divided into three mutually exclusive educational categories. The No College Degree group included women who had attended at least grade school, had attended or graduated high school, and/or had attended some college. The second category, College Degree, included the women who had graduated from college. The third category, Graduate Attendance, comprised women who had attended any graduate school or completed a graduate degree.

Cycle cancellation was defined as having stopped treatment after starting ovarian stimulation before egg retrieval. Reasons for cycle cancellation were grouped into three categories: [1] baseline cancellation—cysts, follicles, fibroids, did not suppress; [2] poor stimulation response; and [3] other/not specified. The peak estradiol level was defined as the highest estradiol level achieved during the IVF cycle before the human chorionic gonadotropin (hCG) trigger. For those who had at least one embryo transferred but experienced no detectable rise in hCG, the outcome was defined as "implantation failure." Chemical pregnancy was defined as a detectable hCG level after embryo transfer without observation of a gestational sac on ultrasound. Spontaneous abortion (SAB) was defined as ultrasound findings of pregnancy, including a yolk sac or fetal heartbeat, with no further pregnancy progression. Live birth was defined as delivery of a viable infant.

Multivariable linear regression was performed to quantify the relation between the woman's education and her peak estradiol level. Multivariable logistic regression was performed to quantify the relation between the woman's education and the dichotomous outcomes of cycle cancellation, implantation failure, chemical pregnancy, SAB, and live birth. If adding a covariate to the model changed the estimated effect of a woman's education on cycle outcome by $10 \%$ or more, the covariate was considered a confounder of the relation between the woman's educational status and IVF outcome and was included in the final multivariable models (5). The peak estradiol level was not included in these models a priori as it is hypothesized to be on the causal pathway between a woman's education and IVF outcome. Of the covariates tested (including stimulation protocol, cigarette-smoking status, physical activity, parity, and history of prior SAB), only the woman's age at cycle start ( $\$ 4$ years, 35 to 39 years, $\geq 40$ years) and the clinic (three sites) were included in the logistic regression models. Age, body mass index (BMI, <18.5, 18.5-24.9, 25.0-29.9, 30.0-39.9, and $\geq 40 \mathrm{~kg} /$ $\mathrm{m}^{2}$ ), and clinic site were included in the multivariable linear regression model addressing peak estradiol levels. $P<.05$ was considered statistically significant. 
Of the 2,569 women in the study population, 9 had attended grade school only and were included with the 570 women who had completed high school in the No College Degree category; 1,105 women were college graduates, and 885 had attended or completed graduate school (Table 1). Women with graduate school participation comprised $33 \%, 42 \%$, and $25 \%$ of the clinic population at each of the three sites. The mean age increased with increasing education category $(34.4,35.1$, and 36.2 years, respectively, with a difference of $<2$ years between the first and third category), and mean BMI decreased with increasing educational level $\left(25.9,24.0\right.$, and $23.0 \mathrm{~kg} / \mathrm{m}^{2}$, respectively). Although most patients were nonsmokers, the majority of current smokers were in the No College Degree category, which is consistent with the greater prevalence of smoking that is associated with lower educational attainment in the general population (6). Histories of prior pregnancy and of SAB were similar across education categories (data not shown).

The majority of patients (73.3\%) underwent IVF stimulation with a down-regulation protocol, and this was consistent across educational categories. When patients were stratified by down-regulation versus flare stimulation protocol, the numbers were too small to quantify within-strata effects.

Among each education level of those with a cancelled cycle, $80 \%$ cancelled due to poor stimulation response. There was a greater proportion of Unspecified Reason indicated in the No College Degree group (16\% vs. $10 \%$ for the other two groups). After adjusting for age and clinic, the odds of cycle cancellation at any point before egg retrieval were $40 \%$ lower among those with an undergraduate degree and $48 \%$ lower among those with a graduate degree compared with women in the No College Degree group ( $P=.001$, test for linear trend). More specifically, among the never-smokers, college graduates and graduate school attendees had 35\% lower odds of cycle cancellation as compared with women with no college degree. Among ever-smokers (past and current), compared with the No College Degree group, the college graduates had $46 \%$ lower odds and women who attended graduate school had $68 \%$ lower odds of cycle cancellation. However, the odds of cancellation were not statistically significantly different among these groups ( $P=.18$, test for heterogeneity). Although there is evidence that cigarette smoking is associated deleteriously with fertility (7) and IVF outcomes (8), adjustment of smoking (never vs. ever) did not alter the observed relation between educational attainment and cycle cancellation.

After we adjusted for age, BMI, and clinic site, the patients with a graduate school education had statistically significantly higher peak estradiol levels than the patients in the No College Degree group ( $P=.02$, test for linear trend). Among women who had an embryo transfer, no statistically significant difference was observed across education groups for any other IVF outcome. For analysis of the association with live birth, we additionally included all cycle starts; similarly, no association with educational attainment was found.

We observed greater odds of cycle cancellation among the women with lower educational attainment, irrespective of age, which suggest that education itself may impact successful progression to egg retrieval. For the women who progressed to egg retrieval, a woman's educational level was also correlated with her peak estradiol level, which may suggest a relation between a woman's education level and comprehension of the cycle instructions. Among women who had an embryo transfer, there was no statistically significant difference across education groups or any subsequent IVF outcome. A separate analysis was performed to include all cycle starts, and similarly no difference in IVF outcomes was found.

Since the close of study enrollment in 2003, the availability of nursing support and pharmaceutical on-call services have increased at most fertility clinics. Furthermore, patient materials are available through lay support groups, Internet blogs, and even on YouTube on 
how to administer the medications, although these sources have not been reviewed and affirmed by clinicians. The increase in available information allows for the potential to reduce differences in the influence of a woman's education on her cycle outcomes by clinic site. However, with the availability of expert-reviewed and nonreviewed material comes the opportunity for misinformation and medication administration error. As the major finding of this study was a correlation between cycle cancellation and lower educational level, this suggests that improved training for proper home-based administration of stimulation regimens may lower the odds of cycle cancellation for patients who have less than a graduate-level education. Furthermore, a targeted educational process may even improve cancellation rates across all educational backgrounds. There may be potential for maximizing IVF treatment outcome with improved and standardized educational and supportive tools.

We conclude that a woman's educational level may impact attainment of sufficient peak estradiol levels and successful progression to oocyte retrieval. After that point, IVF outcomes were not associated with a woman's education. Further studies should evaluate compliance to treatment protocols by educational level.

\section{Acknowledgments}

Supported by National Institutes of Health grant HD32153.

\section{REFERENCES}

1. Ivanans T. Effect of maternal education and ethnic background on infant development. Arch Dis Child. 1975; 50:454-457. [PubMed: 1147687]

2. Ponjaert-Kristoffersen I, Bonduelle M, Barnes J, Nekkebroeck J, Loft A, Wennerholm UB, et al. International collaborative study of intracytoplasmic sperm injection-conceived, in vitro fertilization-conceived, and naturally conceived 5-year-old child outcomes: cognitive and motor assessments. Pediatrics. 2005; 115:e283-e289. [PubMed: 15741353]

3. Shevell T, Malone FD, Vidaver J, Porter TF, Luthy DA, Comstock CH, et al. Assisted reproductive technology and pregnancy outcome. Obstet Gynecol. 2005; 106:1039-1045. [PubMed: 16260523]

4. Morris SN, Missmer SA, Cramer DW, Powers RD, McShane PM, Hornstein MD. Effects of lifetime exercise on the outcome of in vitro fertilization. Obstet Gynecol. 2006; 108:938-945. [PubMed: 17012457]

5. Greenland S. Modeling and variable selection in epidemiologic analysis. Am J Public Health. 1989; 79:340-349. [PubMed: 2916724]

6. Garrett BE, Dube SR, Trosclair A, Caraballo RS, Pechacek TF. Cigarette smoking-United States, 1965-2008. MMWR Surveill Summ. 2011; 60(Suppl):109-113. [PubMed: 21430635]

7. Dechanet C, Anahory T, Mathieu Daude JC, Quantin X, Reyftmann L, Hamamah S, et al. Effects of cigarette smoking on reproduction. Hum Reprod Update. 2011; 17:76-95. [PubMed: 20685716]

8. Waylen AL, Metwally M, Jones GL, Wilkinson AJ, Ledger WL. Effects of cigarette smoking upon clinical outcomes of assisted reproduction: a meta-analysis. Hum Reprod Update. 2009; 15:31-44. [PubMed: 18927070] 
TABLE 1

Women's characteristics and in vitro fertilization (IVF) cycle outcome by education category among 2,569 women commencing their first treatment cycle.

\begin{tabular}{|c|c|c|c|}
\hline Characteristic & $\begin{array}{c}\text { No college degree } \\
(n=579)\end{array}$ & $\begin{array}{l}\text { College degree } \\
\quad(n=1,105)\end{array}$ & $\begin{array}{c}\text { Graduate school } \\
\text { attendance }(n=885)\end{array}$ \\
\hline Age (y), mean (SD) & $34.4(4.5)$ & $35.1(4.2)$ & $36.2(4.1)$ \\
\hline BMI $\left(\mathrm{kg} / \mathrm{m}^{2}\right)$, mean $(\mathrm{SD})$ & $25.9(5.8)$ & $24.0(4.9)$ & $23.0(3.9)$ \\
\hline Current smoker, $\mathrm{N}(\%)$ & $109(18.9)$ & $48(4.3)$ & $20(2.3)$ \\
\hline Down-regulation, $\mathrm{N}(\%)$ & $419(72.4)$ & 805 (72.9) & $658(74.4)$ \\
\hline Embryos transferred, mean (SD) & $2.9(1.2)$ & $2.9(1.2)$ & $3.1(1.3)$ \\
\hline \multicolumn{4}{|l|}{ Outcome } \\
\hline Cycle cancellation, $\mathrm{N}(\%)$ & $64(11.1)$ & $80(7.2)$ & $61(6.9)$ \\
\hline OR $(95 \% \mathrm{CI})^{a}$ & 1.00 (Referent) & $0.60(0.42-0.85)$ & $0.52(0.36-0.76)$ \\
\hline Peak $E_{2}(\mathrm{pg} / \mathrm{mL})$, mean $(\mathrm{SD})$ & $1,535.0(904.1)$ & $1,588.6(945.6)$ & $1,719.0(950.2)$ \\
\hline Adjusted peak $\mathrm{E}_{2}$ mean $\mathrm{pg} / \mathrm{mL}(P \text { value })^{b}$ & $1,465.1$ (Referent) & $1,482.9(.90)$ & $1,599.3(.02)$ \\
\hline Chemical pregnancy, $\mathrm{N}(\%)$ & $37(15.9)$ & $81(18.5)$ & $68(18.5)$ \\
\hline $\mathrm{OR}(95 \% \mathrm{CI})^{a}$ & (Referent) & $1.12(0.73-1.73)$ & $1.07(0.68-1.68)$ \\
\hline $\mathrm{SAB}, \mathrm{N}(\%)$ & $31(15.9)$ & $41(11.5)$ & $46(15.4)$ \\
\hline OR $(95 \% \mathrm{CI})^{a}$ & (Referent) & $0.61(0.37-1.02)$ & $0.76(0.46-1.28)$ \\
\hline Live birth, $\mathrm{N}(\%)^{c}$ & $163(28.2)$ & $313(28.3)$ & $248(28)$ \\
\hline OR $(95 \% \mathrm{CI})^{a}$ & (Referent) & $1.11(0.88-1.39)$ & $1.23(0.96-1.57)$ \\
\hline
\end{tabular}

Note $: \mathrm{BMI}=$ body mass index $; \mathrm{CI}=$ confidence interval $; \mathrm{E}_{2}=$ estradiol $; \mathrm{OR}=$ odds ratio $; \mathrm{SAB}=$ spontaneous abortion; $\mathrm{SD}=$ standard deviation .

${ }^{a}$ Multivariable logistic regression model adjusted for age at cycle start and clinic site.

${ }^{b}$ Multivariable linear regression model adjusted for age, body mass index, and clinic site.

${ }^{c}$ Live birth among all cycle starts. 